\title{
Introduction: Assessment of Skeletal Health-Beyond Bone Density
}

\author{
Susan B. Broy ${ }^{1}$
}

Published online: 10 February 2016

(C) Springer Science+Business Media New York 2016

Keywords Osteoporosis - Dual-energy X-ray absorptiometry · Bone strength - Bone density · Fracture risk - Vertebral fracture assessment . Trabecular bone score $\cdot$ Finite element analysis $\cdot$ Sarcopenia $\cdot$ Body composition

Areal BMD (aBMD) measured by dual-energy X-ray absorptiometry (DXA) is the current standard for the diagnosis and management of patients with osteoporosis and is the basis for the World Health Organization (WHO) diagnostic criteria [1]. However, most patients who fracture do not have osteoporosis by WHO criteria [2-4] because the majority of individuals are in the osteopenic range. Data from the OFELY study (Table 1) illustrate this phenomenon: although patients with osteoporosis have the highest risk of fracture $(37 \%$ 10 year risk), the majority of patients with fracture $(61 \%)$ are in the osteopenic or normal range [3]. Although $60-80 \%$ of bone strength can be attributed to BMD [5-7], there are other factors that contribute to fracture risk and should be considered when deciding who should be treated. Clinical risk factors are important, and FRAX ${ }^{\circledR}$ (fracture risk assessment tool developed by WHO) is now routinely used to quantify the effect of these risk factors on fracture risk. In addition, other qualities of bone such as size and shape (macroarchitecture), microarchitecture and intrinsic material properties are important in determining bone strength and fracture risk. Although we have made major advances in understanding the role of these other factors in bone strength from bone biopsies and advanced

Susan B. Broy

Broysusan@gmail.com

1 Department of Medicine, Rosalind Franklin School of Medicine, Chicago Medical School, 3333 Green Bay Road, North Chicago, IL, USA imaging procedures using quantitative computed tomography and magnetic resonance imaging, until now there have been no measures that could be used in clinical practice.

There are now several non-BMD imaging techniques that can be used to improve fracture risk prediction in clinical practice. This includes parameters that can be obtained from DXA scans: vertebral fracture assessment (VFA), trabecular bone score (TBS), body composition (for the diagnosis of sarcopenia) and hip structural analysis (HSA). Finite element analysis (FEA) is an engineering technique that can be applied to quantitative computed tomography (QCT) images to estimate bone strength. This issue reviews the use of these modalities for the assessment of skeletal health beyond bone density.

\section{Vertebral Fracture Recognition}

Although vertebral fractures are the most common osteoporotic fracture and are associated with a major risk of future osteoporotic fractures [8], they are often not recognized. Dr. Vokes reviews the importance of vertebral fractures and describes methods for diagnosis via standard imaging techniques (X-ray, CT, MRI) as well as vertebral fracture assessment by DXA (VFA). She discusses the controversies regarding diagnostic criteria for fracture and presents International Society for Clinical Densitometry (ISCD) positions on the indications for spinal imaging.

\section{Trabecular Bone Score}

Trabecular bone score is a new US Food and Drug Association (FDA) approved software program that can be applied to DXA scans. TBS assesses the texture of spinal 
Table 1 Prospective study of 668 postmenopausal women in France (OFELY)

\begin{tabular}{llll}
\hline WHO criteria by aBMD & Total $N(\%$ of total $)$ & $\begin{array}{l}10 \text {-year fx } \\
\text { incidence }(\%)\end{array}$ & $\begin{array}{l}N \text { with fracture } \\
(\% \text { of total })\end{array}$ \\
\hline Osteoporosis & $142(21 \%)$ & 37 & $52(39 \%)$ \\
Osteopenia & $322(48 \%)$ & 21 & $67(50 \%)$ \\
Normal BMD & $204(30 \%)$ & 7 & $15(11 \%)$ \\
\hline
\end{tabular}

For the entire population, there was a $20 \%$ incidence of fracture over 10 years. Most fracture patients (61\%) did not have osteoporosis by WHO criteria

WHO World Health Organization, $a B M D$ areal bone mineral density, $N$ number, $f x$ fracture bone from a DXA image-a measurement that is associated with bone microarchitecture. Drs. Binkley and Leslie review the technique of TBS and the in vivo and in vitro evidence that TBS is associated with bone strength and microarchitecture. They then review the clinical studies showing a strong association between TBS and fracture risk. Finally, they discuss the clinical utility of TBS including a method to adjust FRAX calculations based on TBS scores and the use of TBS in specific clinical situations including diabetics and glucocorticoid users.

\section{Finite Element Analysis}

Osteoporosis is a disease of "compromised bone strength predisposing to an increased risk of fracture" [9]. Since a direct measurement of bone strength in vivo is not possible, BMD has been used as a surrogate. However, a direct measurement would be preferable. Finite element analysis is an engineering method that can be used to estimate the strength of a complicated structure such as bone and its response to various loads. Dr. Engelke et al. describe the basic technique and limitations of FEA applied to QCT images of the hip, spine and radius, review precision and validation studies and discuss the potential clinical utility of FEA.

\section{Sarcopenia}

Fractures occur when the load on bone exceeds its strength. Imaging procedures discussed in previous articles assess bone strength, but it is also important to consider the effect of increasing loads (particularly falls) on fracture risk. Sarcopenia is common in our elderly population and can contribute to falls and fractures. Dr. Levinger et al. present the evidence that sarcopenia is associated with falls and fractures, discuss the interaction between bone and muscle, review the diagnostic criteria for sarcopenia and sarcopenic obesity and conclude that a sarcopenia evaluation should be a routine part of an osteoporosis management program.

In a related article, Dr. Shepherd describes how body composition analysis by DXA can be used to diagnose sarcopenia. He describes the general technique of body composition analysis, parameters that can be measured, and shows examples of scans in a mildly obese but otherwise healthy male and a male with sarcopenic obesity.

\section{Clinical Vignettes}

The final article by Dr. Lewiecki presents a series of clinical cases illustrating how measurements discussed in this special issue might be used in clinical practice.

\section{ISCD Position Development Conference on Fracture Prediction Beyond BMD}

An ISCD position development conference was held in Chicago in February 2015 to review the role of many of these new modalities including TBS, FEA and HSA in clinical care. Several task forces presented medical evidence to a panel of experts in the assessment of skeletal health who then discussed and voted on official positions for the use of these techniques. The methodology of this process and the final approved ISCD Official Positions are presented in an executive summary [10]. The expert panel concluded that although there was good evidence that TBS, FEA and hip axis length were associated with fracture risk, they could not be used for diagnosis. However, they do have the potential to improve fracture risk prediction. In particular, TBS "can be used in association with FRAX and BMD to adjust FRAX probability of fracture in postmenopausal women and older men" [10].

\section{Conclusion}

In the absence of fracture, BMD is the best predictor of future fracture. However, BMD alone cannot discriminate between those who will or will not sustain a fragility fracture as most fracture patients do not have osteoporosis by BMD criteria. Recent major advances in our knowledge of bone physiology and the development of new imaging techniques have made it possible to assess skeletal health 
and improve fracture risk prediction in clinical patients. Although these new technologies will not replace the use of BMD measurements, they may be helpful in making clinical decisions in borderline patients. Further research is needed to clarify their role in the clinical management of patients with osteoporosis.

\section{Compliance with Ethical Standards}

Conflict of interest Susan B. Broy has no conflict of interest.

Animal/Human Studies This article does not include any studies with human or animal subjects performed by the author.

\section{References}

1. WHO. Assessment of fracture risk and its application to screening for postmenopausal osteoporosis. Geneva: World Health Organization; 1994.

2. Stone KL, Seeley DG, Lui L, et al. BMD at multiple sites and risk of fracture of multiple types: long-term results from the Study of Osteoporotic Fractures. J Bone Miner Res. 2003;18(11):1947-54.
3. Sornay-Rendu E, Munoz F, Garnero P, et al. Identification of osteopenic women at high risk of fracture: the OFELY study. J Bone Miner Res. 2005;20(10):1813-9.

4. Schuit SC, van der Klift M, Weel AE, et al. Fracture incidence and association with bone mineral density in elderly men and women: the Rotterdam Study. Bone. 2004;34(1):195-202.

5. Moro M, Hecker AT, Bouxsein ML, Myers ER. Failure load of thoracic vertebrae correlates with lumbar bone mineral density measured by DXA. Calcif Tissue Int. 1995;56(3):206-9.

6. Cheng XG, Nicholson PH, Boonen S, Low G. Prediction of vertebral strength in vitro by spinal bone densitometry and calcaneal ultrasound. J Bone Miner Res. 1997;12(10):1721-8.

7. Bousson V, Le Bras A, Roqueplan F, et al. Volumetric quantitative computed tomography of the proximal femur: relationships linking geometric and densitometric variables to bone strength. Role for compact bone. Osteoporos Int. 2006;17:855-64.

8. Klottzbuecher CM, Ross PD, Landsman PB, et al. Patients with prior fractures have an increased risk of future fractures: a summary of the literature and statistical synthesis. J Bone Miner Res. 2000;15(4):721-39.

9. NIH Consensus Development Panel. Osteoporosis prevention, diagnosis, and therapy. JAMA. 2001;285(6):785-95.

10. Shepherd JA, Schousboe JT, Broy SB, Engelke K, Leslie WD. Executive summary of the 2015 ISCD Position Development Conference on Advanced Measures from DXA and QCT: fracture prediction beyond BMD. J Clin Densitom. 2015;18(3):274-86. 